\title{
Validade de construto da versão brasileira do Inventário Espectral de Externalização: evidências a partir de uma amostra de estudantes universitários
}

\author{
Construct validity of the Brazilian version of the Externalizing Spectrum Inventory: evidence from a university \\ student sample
}

\author{
Hudson Wander de Carvalho ${ }^{1}$, Christopher John Patrick², Roberto Frederick Krueger ${ }^{3}$, Kristian Erik Markon ${ }^{4}$, \\ Ângela Maria Vieira Pinheiro 5
}

1 Universidade Federal de São Paulo (Unifesp).

2 Florida State University (FSU).

3 University of Washington (UW).

4 University of lowa.

5 Universidade Federal de Minas Gerais (UFMG).

Recebido: 2/9/2009 - Aceito: 22/10/2009

\begin{abstract}
Resumo
Contexto: Externalização é uma dimensão das diferenças individuais que dá substrato e unifica traços impulsivos e agressivos de personalidade a transtornos mentais relacionados ao uso de substância e à conduta antissocial. Objetivos: $\mathrm{O}$ presente estudo objetivou apresentar indicadores de validade de construto da versão brasileira do Inventário Espectral de Externalização. Método: Trata-se de um estudo psicométrico de orientação correlacional. Foram utilizadas duas técnicas clássicas de representação de construto: análise exploratória de fatores comuns e análise de consistência interna. A amostra consistiu de 258 participantes de ambos os sexos, provenientes de diferentes cursos universitários de duas instituições públicas do estado de Minas Gerais. Resultados: A análise fatorial resultou em um modelo de um fator de segunda ordem (Externalização) com três fatores de primeira ordem (dimensões de agressão, de uso de substância e de impulsividade/alienação). Os coeficientes alfa mostraram-se bastante elevados, com índices acima de 0,9 tanto para os três fatores de primeira ordem quanto para o fator geral. Conclusão: Combinados, os resultados trazem evidências de validade e de fidedignidade para a versão brasileira do Inventário Espectral de Externalização no que tange à sua aplicabilidade a estudantes universitários.
\end{abstract}

Carvalho HW, et al. / Rev Psiq Clín. 2010;37(5):206-11

Palavras-chave: Inventário Espectral de Externalização, psicometria, psicopatologia, personalidade.

\begin{abstract}
Background: Externalizing is a dimension of individual differences that undergirds and unites aggressive and impulsive personality traits to psychopathological disorders related to substance use and antisocial behavior. Objectives: The present study aims to establish parameters of construct validity for the Brazilian version of the Externalizing Spectrum Inventory. Method: This is a psychometrical correlacional study design. Two classical techniques of construct representation were implemented: unweighted least squares exploratory factor analysis and internal consistency analysis. The sample consisted of 258 participants of both sexes, from different undergraduate majors in two public universities in the state of Minas Gerais. Results: As expected in theoretical grounds, common factor analysis revealed a one second-order factor (externalizing dimension) with three subfactor structure (substance, antisocial and impulsivity/alienation dimensions). The alfa coefficients were very high, with indexes greater than 0,9 for the three subfactor as well as for the general factor. Discussion: Taken together, results bring evidences of validity and reliability of the Brazilian version of the Externalizing Spectrum Inventory in regard to it applicability in university students.
\end{abstract}

Carvalho HW, et al. / Rev Psiq Clín. 2010;37(5):206-11

Keywords: Externalizing Spectrum Inventory, psychometrics, psychopathology, personality.

\section{Introdução}

Denomina-se taxonomia o procedimento científico de classificação de elementos e fenômenos naturais, assim como os métodos envolvidos para tal fim. No que tange à Psiquiatria e à Psicologia médica, a taxonomia deve ser compreendida como uma área da ciência que objetiva a construção de um sistema válido de classificação para os transtornos mentais de maneira a organizá-los com base em pressupostos teóricos ${ }^{1}$. Todavia, os sistemas dominantes de classificação dos transtornos mentais são pretensamente ateóricos ${ }^{2,3}$, sendo, desse modo, puramente descritivos. Além disso, a taxonomia psiquiátrica tem se mostrado um tópico altamente controverso ${ }^{4-7}$, de modo que diferentes modelos taxonômicos ${ }^{8-10}$ e definições para o construto transtorno mental7,11-13 têm sido propostos com o objetivo explícito de superação paradigmática.
Dentre os diferentes modelos taxonômicos encontrados na literatura psiquiátrica e psicológica, um modelo dimensional de base biológica denominado de Modelo Hierárquico de Internalização/ Externalização tem recebido especial ênfase tanto em pesquisas empíricas ${ }^{14-17}$ quanto teóricas ${ }^{8-10,18-20}$, que o destacam como um norteador para o desenvolvimento da quinta edição do Manual Estatístico e Diagnóstico de Transtornos Mentais (DSM-V) da Associação Americana de Psiquiatria.

\section{Emergência do Modelo Hierárquico de Internalização/ Externalização}

A emergência do Modelo Hierárquico de Internalização/Externalização se deu com o desenvolvimento de estudos epidemiológicos de base populacional cujo objetivo central era o exame da estrutura 
latente de transtornos mentais relacionados ao uso de substâncias, à conduta antissocial, à depressão, à ansiedade e à fobia, por meio da aplicação de modelagem por equações estruturais ${ }^{15-17}$. Esses estudos indicaram que o padrão de coocorrência entre os citados transtornos mentais pode ser explicado por dois fatores correlacionados denominados de Internalização e de Externalização. Enquanto Internalização explicou a covariância entre transtornos depressivos, de ansiedade e fóbicos, Externalização ocupou-se da variância comum entre os transtornos relacionados à conduta antissocial e ao uso de substâncias. Recentemente, o Modelo Hierárquico de Internalização/ Externalização foi replicado com sucesso e amplamente discutido em um estudo metanalítico9.

Investigações posteriores revelaram que traços de personalidade ligados à instabilidade afetiva e ao controle de impulsos também seriam indicadores latentes das dimensões de Internalização e de Externalização, respectivamente21,22. Adicionalmente, há evidências que sugerem que ambas as dimensões são amplamente herdadas ${ }^{14,23}$ e, recentemente, um modelo endofenotípico de base neurofisiológica foi conjecturado 24 .

Assim, a definição dos construtos se ampliou de modo a abranger tanto síndromes psicopatológicas quanto traços de personalidade. Internalização e Externalização devem, portanto, ser definidas como dimensões altamente hereditárias de vulnerabilidade psicopatológica que explicam as associações entre agrupamentos de traços de personalidade e transtornos mentais, unificando-os por meio de um modelo taxonômico conciso, empiricamente fundamentado e de base psicobiológica.

A adoção do Modelo Hierárquico de Internalização/Externalização traz contribuições importantes para o entendimento e para o status científico tanto da taxonomia psiquiátrica quanto da taxonomia da personalidade, por três razões principais. Primeiramente, o Modelo Hierárquico de Internalização/Externalização organiza seus elementos com base em seus determinantes etiológicos comuns, evoluindo, desse modo, de um modelo puramente descritivo - como os sistemas de classificação DSM e CID - para um de base teórico-explicativa. Em segundo lugar, o modelo unifica e explica a relação entre construtos centrais e entendimento do psiquismo, como personalidade e psicopatologia, que, apesar de frequentemente associados em pesquisas empíricas ${ }^{25-27}$, apresentam desenvolvimentos teóricos e repercussões práticas bastante independentes ${ }^{28}$. Em terceiro e último lugar, o modelo soluciona uma série de impasses práticos e conceituais inerentes aos sistemas classificatórios dominantes, como em relação à controversa definição dos transtornos mentais como fenômenos dimensionais ou discretos ${ }^{18,19}$, ou quanto ao significado da comorbidade psiquiátrica ${ }^{9}$.

Recentemente, o espectro da Externalização foi delineado por meio de um estudo de orientação psicométrica ${ }^{29}$. Esse estudo culminou no desenvolvimento e na validação do Inventário Espectral de Externalização para o contexto norte-americano, cujos procedimentos metodológicos e resultados serão descritos a seguir.

\section{Inventário Espectral de Externalização: origem, desenvolvimento e procedimentos de validação de construto}

A origem do Modelo Espectral de Externalização situa-se em um estudo de genética comportamental desenvolvido por Krueger et al. ${ }^{23}$. Os autores analisaram a estrutura fatorial de dados diagnósticos (transtorno de conduta, transtorno de personalidade antissocial, dependência de álcool e drogas) e psicométricos (dimensão de controle de impulsos) via análise fatorial confirmatória em uma ampla amostra de gêmeos monozigóticos e dizigóticos de ambos os sexos. Os resultados indicaram que uma dimensão geral (fator de Externalização) explicou mais do que $80 \%$ da variância genética aditiva, enquanto variáveis ambientais não compartilhadas explicaram a maior parte da variância genética residual. O referido estudo avançou na conceitualização do Modelo Espectral de Externalização por ter demonstrado que uma única dimensão hereditária de vulnerabilidade contribui para o desenvolvimento de diferentes transtornos mentais e traços de personalidade relacionados à tríade impulsividade, uso de substâncias e conduta antissocial.

Subsequentemente, Krueger et al. ${ }^{29}$ desenvolveram um estudo psicométrico que avaliou a dimensão de Externalização sob uma ótica taxométrica. Para tanto, foi elaborado um conjunto amplo de itens de autopreenchimento baseados em construtos candidatos à constituição do espectro da Externalização. O conteúdo dos itens foi determinado por meio de um processo interativo que envolveu diferentes fontes de informação (pesquisas, critérios diagnósticos, testes psicométricos de personalidade e definições operacionais propostas pela equipe de pesquisa). Análises psicométricas e estruturais foram aplicadas em três etapas de coleta de dados com amostras independentes, a fim de refinar os itens, identificar agrupamentos unidimensionais e delinear um modelo estrutural que acomodasse bem os dados. Esse procedimento resultou em 23 escalas/construtos que compõem simultaneamente o Inventário Espectral de Externalização e o espectro conceitual da dimensão de Externalização. A tabela 1 pormenoriza o domínio conceitual e o número de itens referentes a cada escala que compõe a versão final do Inventário Espectral de Externalização.

Tabela 1. Descrição do nome, do número de itens e do conteúdo referente a cada escala que compõe o Inventário Espectral de Externalização

\begin{tabular}{|c|c|c|}
\hline Escala & № de itens & Conteúdo \\
\hline Agressividade destrutiva & 19 & Vandalismo e piromania \\
\hline Agressividade física & 21 & $\begin{array}{l}\text { Uso de armas, ruminação } \\
\text { agressiva, ameaças físicas }\end{array}$ \\
\hline Agressividade relacional & 19 & Boataria, injúria, insultos \\
\hline Alienação & 09 & $\begin{array}{l}\text { Sentir-se manipulado, usado e } \\
\text { maltratado }\end{array}$ \\
\hline Busca de excitação & 18 & Busca de emoções fortes \\
\hline Confiança & 23 & $\begin{array}{l}\text { Conscienciosidade, } \\
\text { responsabilidade }\end{array}$ \\
\hline Controle/planejamento & 11 & $\begin{array}{l}\text { Planejamento, prudência e } \\
\text { autocontrole }\end{array}$ \\
\hline Empatia & 31 & $\begin{array}{l}\text { Remorso, preocupação e } \\
\text { solidariedade }\end{array}$ \\
\hline Externalização da culpa & 14 & $\begin{array}{l}\text { Negar responsabilidade sobre } \\
\text { atos, sentimentos de ser } \\
\text { injustamente culpado }\end{array}$ \\
\hline Fraude & 14 & $\begin{array}{l}\text { Mentir e fingir para receber } \\
\text { benefícios }\end{array}$ \\
\hline Furto & 15 & $\begin{array}{l}\text { Furto, arrombamento, mentir para } \\
\text { a polícia }\end{array}$ \\
\hline Honestidade & 15 & Dizer a verdade, integridade \\
\hline Impulsividade problemática & 20 & $\begin{array}{l}\text { Impulsividade relacionada a } \\
\text { consequências negativas }\end{array}$ \\
\hline Irresponsabilidade & 25 & $\begin{array}{l}\text { Não comparecimento a } \\
\text { compromissos sociais e laborais }\end{array}$ \\
\hline Problemas com álcool & 30 & $\begin{array}{l}\text { Uso abusivo e dependente de } \\
\text { álcool }\end{array}$ \\
\hline Problemas com drogas & & $\begin{array}{l}\text { Uso abusivo e dependente de } \\
\text { drogas }\end{array}$ \\
\hline Problemas com maconha & 18 & $\begin{array}{l}\text { Uso abusivo e dependente de } \\
\text { maconha }\end{array}$ \\
\hline Propensão ao tédio & 25 & $\begin{array}{l}\text { Sentir-se chateado ou aborrecido } \\
\text { facilmente }\end{array}$ \\
\hline Rebeldia & 15 & $\begin{array}{l}\text { Tendência de violar regras e de } \\
\text { desobedecer a ordens }\end{array}$ \\
\hline Urgência impaciente & 12 & $\begin{array}{l}\text { Impaciência diante de } \\
\text { necessidades }\end{array}$ \\
\hline Uso de álcool & 23 & Quantidade de uso de álcool \\
\hline Uso de drogas & 13 & Quantidade de uso de drogas \\
\hline Uso de maconha & 17 & Quantidade de uso de maconha \\
\hline
\end{tabular}


Todas as escalas apresentaram indicadores de fidedignidade substancialmente elevados, de maneira que a maioria alcançou índices $\rho$ superiores a 0.9 . Os resultados da análise fatorial confirmatória evidenciaram que as 23 escalas apresentaram cargas fatoriais substanciais em um fator superordenado (Externalização), e a variância residual de algumas escalas foi explicada por duas dimensões subordenadas independentes. Um dos fatores subordenados foi determinado pela variância residual das escalas de conteúdo antissocial (agressões, honestidade, busca de sensações, rebeldia) e o outro fator, pela variância residual das escalas de problemas com substâncias e de uso de substâncias. A tabela 2 descreve a estrutura fatorial delineada para a versão americana do Inventário Espectral de Externalização (para maior detalhamento, ver Krueger et al. ${ }^{29}$ ).

Tabela 2. Matriz fatorial delineada por Krueger et al.29 para o Inventário Espectral de Externalização. Cargas fatoriais acima de 0.30 estão destacadas, conforme no texto original

\begin{tabular}{l|c|c|c}
\hline \multirow{2}{*}{ Escalas } & \multicolumn{3}{|c}{ Fatores } \\
\cline { 2 - 4 } & Fator geral & $\begin{array}{c}\text { Primeiro } \\
\text { subfator }\end{array}$ & $\begin{array}{c}\text { Segundo } \\
\text { subfator }\end{array}$ \\
\hline Irresponsabilidade & 0.93 & 0.00 & 0.01 \\
\hline Confianças & -0.66 & -0.15 & 0.00 \\
\hline Impulsividade problemática & 0.91 & 0.00 & 0.04 \\
\hline Urgência impaciente & 0.73 & 0.22 & 0.00 \\
\hline Controle e planejamento & 0.73 & -0.07 & 0.00 \\
\hline Furto & 0.87 & 0.00 & 0.13 \\
\hline Alienação & 0.49 & 0.01 & 0.00 \\
\hline Externalização da culpa & 0.51 & 0.24 & 0.00 \\
\hline Agressividade relacional & 0.62 & 0.68 & 0.00 \\
\hline Agressividade destrutiva & 0.65 & 0.55 & 0.00 \\
\hline Agressividade física & 0.74 & 0.41 & 0.00 \\
\hline Empatia & -0.48 & -0.55 & 0.00 \\
\hline Busca de excitação & 0.56 & 0.46 & 0.00 \\
\hline Rebeldia & 0.79 & 0.31 & 0.00 \\
\hline Propensão ao tédio & 0.59 & 0.28 & 0.00 \\
\hline Honestidade & -0.54 & 0.00 & 0.00 \\
\hline Fraude & 0.87 & 0.00 & 0.00 \\
\hline Uso de maconha & 0.73 & 0.00 & 0.61 \\
\hline Problemas com maconha & 0.75 & 0.00 & 0.48 \\
\hline Uso de drogas & 0.79 & 0.00 & 0.49 \\
\hline Problemas com drogas & 0.87 & 0.00 & 0.30 \\
\hline Uso de álcool & 0.45 & 0.00 & 0.36 \\
\hline Problemas com álcool & 0.69 & 0.00 & 0.24 \\
\hline & & & \\
\hline
\end{tabular}

As escalas do Inventário Espectral de Externalização apresentaram distribuição normal e, quando observadas separadamente, demonstraram sensibilidade diferencial no que tange à avaliação de tendências à Externalização em indivíduos com diferentes graus de manifestação. A escala de uso de maconha, por exemplo, mostrouse hábil em diferenciar pessoas que se localizam na distribuição média do traço; já as escalas de problemas com drogas e de agressão destrutiva são mais sensíveis às diferenças individuais localizadas no extremo de gravidade da distribuição do traço. Escalas como busca de excitação se mostraram discriminativas em qualquer grau de manifestação do traço.

Recentemente, o Inventário Espectral de Externalização foi adaptado e validado para um contexto brasileiro ${ }^{30}$. A descrição do procedimento de adaptação do referido instrumento assim como os dados a respeito da consistência interna de suas escalas estão disponibilizados em um trabalho já publicado ${ }^{31}$. Os dados ora apresentados se referem à mesma base de dados que permitiu a realização dos estudos supracitados. O objetivo do presente manuscrito é o de descrever o estudo de validade (validade fatorial) e de confiabilidade (consistência interna) da versão brasileira do Inventário Espectral de Externalização em uma amostra de estudantes universitários mineiros, por meio de procedimentos analíticos exploratórios.

\section{Método}

\section{Participantes}

Participaram voluntariamente do presente estudo 258 estudantes universitários de ambos os sexos (39,1\% de homens). Os participantes eram originários de diferentes instituições universitárias públicas e de diferentes áreas de formação acadêmica, de maneira que 16,7\% eram estudantes da área de saúde (Fisioterapia, Fonoaudiologia e Terapia Ocupacional), 45,7\%, da área de ciências humanas (Antropologia Comunicação Social, Letras e Psicologia) e 37,6\%, da área de artes (Artes Cênicas, Design Gráfico e Design de Produtos). A tabela 3 descreve mais detalhadamente as características da amostra.

Tabela 3. Estatística descritiva da amostra total e dividida por sexo e área de estudo

\begin{tabular}{|c|c|c|c|c|}
\hline \multirow{2}{*}{ Área de estudo } & \multirow{2}{*}{$\mathrm{N}(\%)$} & \multicolumn{2}{|c|}{ Sexo } & \multirow{2}{*}{$\begin{array}{c}\text { Idade } \\
\text { (média e DP) }\end{array}$} \\
\hline & & $\hat{0}$ & $q$ & \\
\hline Saúde & $43(16,7 \%)$ & $5(5 \%)$ & $38(24,2 \%)$ & $23,12(4,767)$ \\
\hline Humanas & $118(45,7 \%)$ & $35(34,7 \%)$ & $83(52,9 \%)$ & $24,16(5,108)$ \\
\hline Artes & $97(37,6 \%)$ & $61(60,4 \%)$ & $36(22,9 \%)$ & $23,84(5,97)$ \\
\hline Total & $258(100 \%)$ & $101(39,1 \%)$ & $157(60,9 \%)$ & $23,49(5,138)$ \\
\hline
\end{tabular}

\section{Instrumento}

O Inventário Espectral de Externalização é um instrumento de autopreenchimento composto por 415 afirmativas que se organizam em 23 escalas. As respostas são mensuradas por uma escala Likert de quatro pontos: verdadeiro; em grande parte verdadeiro; em grande parte falso e falso. O tempo aproximado de sua aplicação em estudantes universitários foi de 50 minutos.

\section{Procedimentos}

A coleta e a tabulação dos dados foram conduzidas por quatro estudantes de graduação do curso de Psicologia da UFMG, que, além de terem concluído as disciplinas obrigatórias de avaliação psicológica, foram devidamente treinados no procedimento delineado para a administração do Inventário Espectral de Externalização. Os dados foram tabulados e analisados por meio da $14^{\text {a }}$ versão do aplicativo SPSS para Windows.

Os participantes preencheram voluntariamente os inventários durante o período letivo, em sala de aula, nos turnos matutino e vespertino. Além disso, nenhuma informação que possibilitasse a identificação dos participantes foi solicitada, garantindo total anonimato e encorajando, desse modo, a veracidade das respostas.

Previamente à coleta de dados, procurou-se obter não somente o consentimento dos diretores das unidades acadêmicas, como também a dos professores, que permitiram a utilização de seu horário de aula para a realização da pesquisa. Ainda, todos os participantes assinaram o Termo de Consentimento Livre e Esclarecido, aprovado pelo Comitê de Ética da UFMG previamente ao início da coleta de dados (Processo ETIC nº 090/06).

\section{Análise dos dados}

Análise exploratória de fatores comuns (AEFC) e análise da consistência interna por meio da estimação do coeficiente alfa foram as técnicas estatísticas escolhidas para avaliar a dimensionalidade/estrutura latente e a consistência interna/homogeneidade do Inventário Espectral de Externalização, respectivamente. As referidas técnicas foram selecionadas em virtude de seu valor para a psicometria no que tange à validade de construto ${ }^{32-34}$. As análises estruturais tiveram 
como base o escore geral obtido pelos participantes nas 23 escalas do Inventário Espectral de Externalização, somando-se a totalidade dos itens referentes a cada faceta.

A AEFC foi efetivada por meio de um estimador denominado análise de quadrados mínimos não ponderados, por causa de sua superioridade estatística em relação a outros métodos de estimação fatorial com dados contínuos e por não apresentar pressupostos ligados à distribuição da amostra ${ }^{35,36}$.

Os fatores de primeira ordem foram estimados por meio de um procedimento de rotação oblíqua denominado de Promax (kappa =4). As correlações entre os fatores foram reparametrizadas como indicadoras de um fator geral de segunda ordem. O número de fatores e o ajuste do modelo estimado foram avaliados com base em indicadores clássicos: presença de cargas multifatoriais; gráfico de scree; análise da tabela de comunalidades e inteligibilidade dos fatores.

A análise da consistência interna teve como base a solução fatorial encontrada para o Inventário Espectral de Externalização. Assim, estimaram-se os coeficientes alfa tanto para os fatores de primeira ordem quanto para o fator geral. A análise de fidedignidade para cada uma das 23 escalas do Inventário Espectral de Externalização está disponibilizada em Carvalho ${ }^{30}$ e Carvalho et al. ${ }^{31}$.

\section{Resultados}

\section{Estrutura fatorial}

Todos os indicadores relativos à adequação da amostra apontam para a presença de variabilidade e multicolinearidade suficientes na matriz de dados para a implementação da AEFC. O coeficiente de Kaiser-Meyer-Olkin (KMO) apresentou magnitude aceitável (0.872), e o teste Bartlett de esfericidade foi significativo $(\mathrm{p}=0.000)$. Os coeficientes obtidos por meio do cálculo da medida de adequação da amostra (MAS) se mostraram acima de 0.7 para todas as escalas, de forma que o índice menos expressivo foi o da escala de problemas com maconha (MAS $=0.785)$ e o mais expressivo, o da escala de busca de excitação (MAS $=0.933$ ).

O teste de scree (autovalores iniciais maiores que uma unidade) e a interpretação da tabela de comunalidades indicaram a presença de quatro fatores de primeira ordem. Todavia, um modelo tetrafatorial produziu uma solução difícil de ser interpretada em virtude da intensa presença de cargas multifatoriais. Além disso, o quarto fator extraído parecia um artefato estatístico, fruto de variância-erro, tanto por não contribuir significativamente para a explicação da variância comum após a rotação $(3,9 \%$ da variância total após rotação) quanto pela dificuldade de compreender o seu significado psicológico. Desse modo, extrai-se um modelo de três fatores correlacionados.

A solução trifatorial se mostrou parcimoniosa, inteligível e com apenas uma carga multifatorial. O primeiro fator explicou a covariância entre as escalas de uso de substâncias, de problemas com substâncias, furto e rebeldia, de maneira que a escala de uso de drogas apresentou a carga mais expressiva (0.973) e a de furto, a menos expressiva (0.377). O segundo fator explicou a covariância entre as escalas de impulsividade e de alienação, e urgência impaciente $(0.890)$ foi a escala que mais fortemente caracterizou o fator e busca de excitação (0.336), a que apresentou a mais fraca associação com ele. $\mathrm{O}$ terceiro fator explicou a covariância entre as escalas que avaliam diferentes tipos de agressão e traços antissociais, de modo que a escala mais associada ao fator foi a de agressão destrutiva (0.867) e a que menor associação apresentou foi a de fraude (0.481). As correlações foram moderadas entre o primeiro e o segundo fator (0.402) e entre o primeiro e o terceiro fator (0.488). Já uma associação entre o segundo e o terceiro fator se mostrou mais substancial (0.65). O primeiro fator foi rotulado de vulnerabilidade ao uso nocivo de substância, o segundo fator, de controle de impulsos e de alienação, e o terceiro fator, de vulnerabilidade antissocial. A tabela 4 traz a matriz fatorial rotada e a porcentagem total de variância explicada pelos fatores, individualmente. Cargas fatoriais acima de 0.32 foram consideradas significativas com base nas recomendações de Costello e Osborne ${ }^{37}$ e, por essa razão, destacadas.
Tabela 4. Matriz fatorial após rotação para uma solução de três fatores correlacionados. Cargas acima de 0.32 e a variância explicada isoladamente por fator estão destacadas

\begin{tabular}{l|c|c|c}
\hline \multirow{2}{*}{ Escalas } & \multicolumn{3}{|c}{ Fatores } \\
\cline { 2 - 4 } & $\begin{array}{c}\text { Fator 1 } \\
36.57 \%\end{array}$ & $\begin{array}{c}\text { Fator 2 } \\
12.44 \%\end{array}$ & $\begin{array}{c}\text { Fator 3 } \\
5.33 \%\end{array}$ \\
\hline Uso de drogas & 0.973 & -0.189 & 0.048 \\
\hline Uso de maconha & 0.950 & -0.130 & -0.136 \\
\hline Problemas com maconha & 0.899 & 0.070 & -0.240 \\
\hline Problemas com drogas & 0.814 & 0.068 & -0.085 \\
\hline Problemas com álcool & 0.549 & 0.063 & 0.197 \\
\hline Uso de álcool & 0.506 & -0.037 & 0.239 \\
\hline Rebeldia & 0.396 & 0.162 & 0.312 \\
\hline Urgência impaciente & -0.111 & 0.890 & -0.143 \\
\hline Impulsividade problemática & 0.109 & 0.846 & -0.066 \\
\hline Controle e planejamento & 0.154 & 0.715 & -0.254 \\
\hline Alienação & -0.147 & 0.683 & 0.009 \\
\hline Propensão ao tédio & -0.105 & 0.661 & 0.061 \\
\hline Externalização da culpa & -0.206 & 0.584 & 0.195 \\
\hline Irresponsabilidade & 0.237 & 0.468 & 0.152 \\
\hline Confiança & 0.188 & 0.373 & 0.183 \\
\hline Busca de excitação & 0.301 & 0.336 & 0.195 \\
\hline Agressividade destrutiva & -0.074 & -0.144 & 0.867 \\
\hline Agressividade relacional & -0.132 & 0.146 & 0.729 \\
\hline Agressividade física & -0.092 & 0.093 & 0.678 \\
\hline Empatia & -0.021 & -0.179 & 0.622 \\
\hline Furto & 0.377 & -0.134 & 0.531 \\
\hline Honestidade & 0.007 & 0.072 & 0.506 \\
\hline Fraude & 0.109 & 0.288 & 0.481 \\
\hline & & & \\
\hline
\end{tabular}

Os fatores extraídos foram redimensionados como indicadores latentes de um fator geral, de segunda ordem. Como esperado, um fator foi suficiente para explicar a covariância entre os fatores de primeira ordem $(58,87 \%$ da variância total), sendo denominado de fator de Externalização. O fator de vulnerabilidade antissocial apresentou a mais intensa associação com o fator geral (0.871), seguido pelo de vulnerabilidade ao uso nocivo de substâncias (0.668) e pelo de controle de impulsos e alienação (0.531). A figura 1 traz uma representação visual da estrutura fatorial modelada: um fator de segunda ordem explica a variância comum entre os três fatores de primeira ordem.

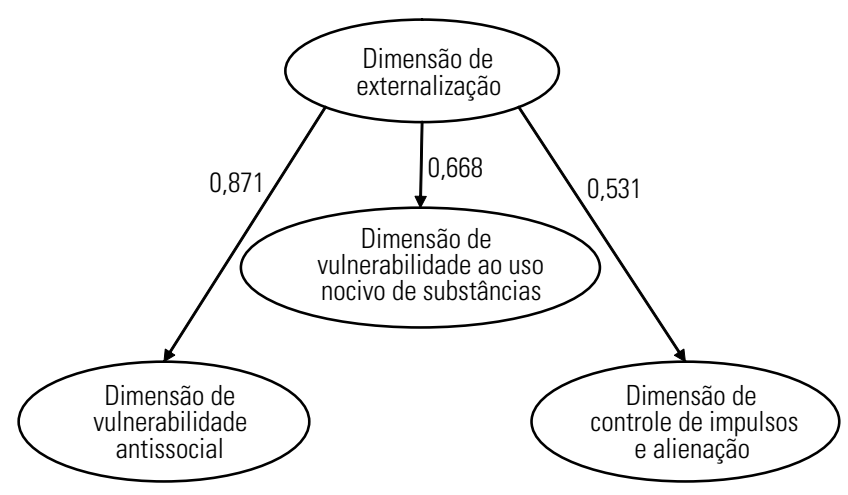

Figura 1. Diagrama do modelo fatorial modelado para a versão brasileira do Inventário Espectral de Externalização. Cargas fatoriais estão destacadas. 


\section{Consistência interna}

Os coeficientes alfa apresentaram magnitude admirável tanto para os fatores de primeira ordem quanto para o fator de segunda ordem. $\mathrm{O}$ fator de vulnerabilidade ao uso nocivo de substâncias apresentou um alfa de 0.975 , o fator de controle de impulsos e alienação apresentou um alfa de 0.967 e o fator de vulnerabilidade antissocial, um alfa de 0.948. O fator de Externalização apresentou um alfa de 0.983. A tabela 5 traz os coeficientes alfa e o número de itens para os fatores extraídos.

Tabela 5. Coeficientes alfa de Cronbach e número total de itens de cada fator extraído para a versão brasileira do Inventário Espectral de Externalização

\begin{tabular}{l|c|c}
\hline Fatores & Coeficiente alfa & Número de itens \\
\hline Fator de substâncias & 0.975 & 139 \\
\hline Fator de personalidade & 0.967 & 144 \\
\hline Fator antissocial & 0.948 & 125 \\
\hline Fator de externalização & 0.983 & 408 \\
\hline
\end{tabular}

Os itens de número 86 (Eu já injetei drogas) e 387 (O meu hábito de beber já me causou problemas com a lei), componentes do fator de vulnerabilidade ao uso nocivo de substâncias, e os itens de número 61 (Eu já usei uma arma em uma briga), 101 (Eu já deixei de pagar a pensão alimentícia), 117 (Eu já comprei coisas usando um cartão de crédito ou seu número roubado), 208 (Eu já usei uma arma para conseguir algo que eu desejava) e 271 (Eu já fui indiciado(a) por agressão), referentes ao fator de vulnerabilidade antissocial, foram automaticamente excluídos da análise de consistência interna por não terem apresentado variabilidade quanto às respostas dadas pelos participantes da pesquisa (100\% de respostas negativas).

\section{Discussão}

Os sistemas de classificação em Psiquiatria e em Psicologia da Personalidade podem ser considerados empreendimentos científicos em processo de construção. As críticas acerca dos modelos dominantes de classificação dos transtornos mentais ${ }^{2,3}$ vão desde o modo como são definidos os transtornos mentais até o perfil ateórico da organização dos manuais diagnósticos-7,12,18. Com o objetivo de superar tais limitações, um modelo taxonômico hierárquico bidimensional, denominado Modelo Hierárquico de Internalização/Externalização, tem sido testado e aperfeiçoado empiricamente em pesquisas tanto empíricas ${ }^{15-17}$ quanto teóricas ${ }^{8,9}$. No que tange à dimensão de Externalização, recentemente, um modelo taxonômico (Modelo Espectral de Externalização) amplo e contínuo, constituído tanto por indicadores psicopatológicos quanto por traços de personalidade, foi delineado por meio de um procedimento psicométrico, resultando na elaboração do Inventário Espectral de Externalização ${ }^{23}$. O presente estudo objetivou avaliar a validade e a fidedignidade desse inventário por meio da aplicação de procedimentos psicométricos clássicos de representação de construto (validação fatorial e análise de fidedignidade/consistência interna) em uma amostra de estudantes universitários do estado de Minas Gerais.

O modelo fatorial delineado resultou em uma estrutura de um fator geral com três fatores de primeira ordem. Conforme as expectativas conceituais, o fator geral foi denominado de fator de Externalização. Os fatores de primeira ordem foram rotulados como vulnerabilidade antissocial, vulnerabilidade ao uso nocivo de substâncias e controle de impulsos e alienação, conforme o seu conteúdo. Adicionalmente, os coeficientes alfa se mostraram bastante elevados, tanto para os fatores de primeira ordem quanto para o fator de segunda ordem.

Combinados, os resultados trazem evidências de validade e de fidedignidade para a versão brasileira do Inventário Espectral de Externalização no que tange à sua aplicabilidade a estudantes universitários. Adicionalmente, o presente estudo contribui para o entendimento da relação latente entre a impulsividade, o uso nocivo de substâncias e a conduta antissocial, por mostrar que a variância comum entre medidas heterogêneas dos referidos construtos pode ser explicada por um fator geral de vulnerabilidade, denominado de fator de Externalização.

Este estudo difere do de Krueger et al..$^{23}$ com relação ao objetivo, ao procedimento de amostragem e às técnicas estatísticas implementadas. Tais diferenças, que serão pormenorizadas a seguir, restringem a comparabilidade entre os dois trabalhos, explicam as diferenças encontradas entre eles e deflagram algumas limitações da presente investigação.

O estudo americano ${ }^{23}$ teve como meta central delinear um modelo taxonômico amplo e quantitativo do espectro da Externalização, sendo o Inventário Espectral de Externalização o resultado de uma pesquisa taxométrica básica, e não a sua meta. Para tanto, itens foram elaborados com o intuito de mensurar os principais elementos constitutivos do referido espectro em uma escala métrica comum que possibilitasse uma eficiente coleta de dados em uma amostra ampla e diversificada. As técnicas estatísticas empregadas - modelagem por equações estruturais e análise do funcionamento diferencial dos itens - permitiram delinear e testar o ajuste de diferentes modelos latentes para o espectro da Externalização por meio de indicadores inferenciais de ajuste de modelo. Ademais, outros indicadores psicométricos - o funcionamento e a precisão de cada uma das escalas que compõe o inventário - foram estimados por meio de métodos psicométricos modernos.

Contrastivamente, o estudo brasileiro apresentou um caráter eminentemente exploratório, cuja meta central residiu na análise psicométrica do Inventário Espectral de Externalização para um contexto específico, o de universitários de Belo Horizonte. Ainda, os procedimentos estatísticos executados se referem ao exame da validade de construto segundo a psicometria clássica. Desse modo, a presente investigação não deve ser posicionada como uma replicação do estudo de Krueger et al.23.

Apesar de evidência empírica ${ }^{38}$ mostrar que diferentes tipos de amostra deflagram estruturas fatoriais substancialmente semelhantes, destaca-se que o caráter homogêneo dos participantes da presente pesquisa pode ter limitado o poder de generalização dos resultados. Tal limitação é deflagrada pela ausência de variabilidade em alguns itens, especificamente aqueles delineados para avaliar manifestações mais graves da dimensão de externalização, pela análise de consistência interna. Por essa razão, enfatizou-se a importância de se desenvolver pesquisas em que as propriedades psicométricas da versão brasileira do Inventário Espectral de Externalização sejam testadas em amostras amplas e casuais, assim como em amostras clínicas, incluindo indivíduos em situação prisional e em tratamento em decorrência do uso de substâncias.

Um importante avanço do presente estudo no que tange à mensuração das diferenças individuais em externalização se refere ao caráter taxonômico amplo do Inventário Espectral de Externalização. As escalas e os itens do inventário representam a operacionalização de todo o espectro da externalização, desde suas manifestações mais suaves, cotidianas e normais às mais graves, raras e psicopatológicas. Nenhum outro instrumento foi construído com o referido objetivo e, portanto, não há outra medida do espectro da externalização, mas apenas instrumentos que avaliam suas facetas isoladamente. Assim, destaca-se o Inventário Espectral de Externalização como o único instrumento avaliativo do espectro da externalização, apresentando várias aplicações possíveis para clínicos e pesquisadores ${ }^{39}$.

\section{Agradecimento}

Ao Conselho Nacional de Desenvolvimento Científico e Tecnológico $(\mathrm{CNPq})$, pelo financiamento.

\section{Referências}

1. Millon T. Classification in psychopathology: rationale, pathology, and standards. J Abnorm Psychol. 1991;100:245-61. 
2. Associação Americana de Psiquiatria. DSM-IV-TR, Manual Diagnóstico Estatístico dos Transtornos Mentais. 4. ed. (texto revisado). Porto Alegre: Artmed; 2002.

3. Organização Mundial de Saúde. CID 10. Classificação Estatística Internacional de Doenças e Problemas Relacionados à Saúde. Porto Alegre: Artmed; 1993.

4. Clark LA, Watson D, Reynalds S. Diagnostic and Classification of Psychopathology: Challenges to the Current System and future Directions. Annu Rev Psychol. 1995;46:121-53.

5. Krueger RF, Watson D, Barlow DH. Introduction to the special section: toward a dimensionally based taxonomy of psychopathology. J Abnorm Psychol. 2005;114:491-3.

6. Widiger TA, Clark LA. Towards DSM-V and the classification of Psychopathology. Psychol Bull. 2005;126:946-62.

7. Widiger TA, Sankis LM. Adult psychopathology: issues and controversies. Annu Rev Psychol. 2000;51:377-404.

8. Krueger RF, Markon KE. Understanding psychopathology: melding behavior genetics, personality, and quantitative psychology to develop an empirically based model. Curr Dir Psychol Sci. 2006;15:113-7.

9. Krueger RF, Markon KE. Reinterpreting comorbidity: a model-based approach to understanding and classifying psychopathology. Annu Rev Clin Psychol. 2006;2:111-33.

10. Watson D. Rethinking mood and anxiety disorders: a quantitative hierarchical model for DSM-V. J Abnorm Psychol. 2005;114:522-36.

11. Wakenfield JC. Disorders as harmful dysfunction: a conceptual critique of DSM-III-R's definition of mental disorder. Psychol Rev. 1992;99:232-47.

12. Wakenfield JC. Diagnosing DSM-IV - Part 1: DSM-IV and the concept of disorder. Behav Res Ther. 1997;102:169-72.

13. Bergner RM. What is psychopathology? And so what? Clinical Psychology: Science and Practice. 1997;4:235-48.

14. Kendler KS, Prescott CA, Myers J, Neale MC. The structure and Environmental risk factors for common psychiatric and substance use disorders in men and women. Arch Gen Psychiatry. 2003;60:929-37.

15. Krueger RF, Caspi A, Moffit TE, Silva PA. The structure and stability of common mental disorders (DSM-III-R): a longitudinal - epidemiological study. J Abnorm Psychol. 1998;107(2):216-27.

16. Krueger RF. The structure of common mental disorders. Arch Gen Psychiatry. 1999;56:921-6.

17. Vollebergh W, Iedema J, Bijl R, de Graaf R, Smit F, Ormel J. The structure and stability of common mental disorders: the NEMESIS study. Arch Gen Psychiatry. 2001;58:597-603.

18. Brown TA, Barlow DH. Dimensional versus categorical classification of mental disorders in fifth edition of the diagnostic and statistical manual of mental disorders and beyond: comment on the special section. J Abnorm Psychol. 2005;114:551-6.

19. Krueger RF, Watson D, Barlow DH. Introduction to the special section: toward a dimensionally based taxonomy of psychopathology. J Abnorm Psychol. 2005;114:491-3.

20. Krueger RF, Markon KE, Patrick CJ, Iacono WG. Externalizing psychopathology and its implications for DSM-V. J Abnorm Psychol. 2005;114:492-3.

21. Krueger RF, McGue M, Iacono WG. The higher-order structure of common DSM mental disorders: internalizing, externalizing, and their connections to personality. Pers Individ Dif. 2001:30:1245-59.
22. Markon KE, Krueger RF, Bouchard TJJ, Gottesman II. Normal and abnormal personality traits: evidence for genetic and environmental relationships in the Minnesota Study of Twins Reared Apart. J Pers. 2002;70:661-93.

23. Krueger RF, Hicks BM, Patrick CJ, Carlson SR, Iacono WG, McGue M. Etiologic connections among substance dependence, antisocial behavior, and personality: modeling the externalizing spectrum. J Abnorm Psychol. 2002;107:216-27.

24. Patrick CJ, Bernat EM. The construct of emotion as a bridge between personality and psychopathology. In: Krueger RF, Tackett JL, editor. Personality and psychopathology. New York: The Guilford Press; 2006, p. 174-209.

25. Costa PT Jr, McCrae RR. Personality disorders and the five-factor model of personality. J Pers Dis. 1990;4:362-71.

26. Costa PT Jr, McCrae RR. The five-factor model of personality and its relevance to personality disorders. J Pers Dis. 1992;6:343-59.

27. Desrichard O, Denarié V. Sensation seeking and negative affectivity as predictors of risky behaviour: a distinction between occasional versus frequent risk-taking. Addict Behav. 2005;30:1449-53.

28. Mathews G, Deary IJ, Whiteman MC. Personality traits. 2. ed. Cambridge: Cambridge University Press; 2003.

29. Krueger RF, Markon KE, Patrick CJ, Benning S, Kramer M. Linking antisocial behavior, substance use and personality: towards a comprehensive quantitative model of adult externalizing spectrum. J Abnorm Psychol. 2007;116:645-66.

30. Carvalho HW. Tradução, adaptação e validação fatorial do Inventário de Externalização para o contexto de universitários mineiros [dissertação não publicada]. Departamento de Pós-Gradução em Psicologia, Universidade Federal de Minas Gerais, Minas Gerais; 2007

31. Carvalho HW, Pinheiro AMV, Patrick CJ, Krueger RF, Markon KE. Tradução, adaptação cultural e análise de consistência interna do Inventário de Externalização. Avaliação Psicológica. 2007;6:217-27.

32. Cronbach LJ, Meehl PE. Construct validity in psychological tests. Psychol Bull. 1955;52:281-302.

33. Pasquali L. Psicometria: teoria dos testes na psicologia e na educação. Petrópolis: Rio de Janeiro: Vozes; 2003.

34. Pasquali L. Princípios de elaboração de escalas psicológicas. Rev Psiq Clín. 1998;25(5):206-13.

35. Briggs NE, MacCallum RC. Recovery of weak common factors by Maximum Likelihood and Ordinary Least Squares estimation. Multivariate Behav Res. 2003;38:25-56.

36. McDonald RP. Factor analysis and related methods. New Jersey: Lawrence Erlbaum Associates; 1985.

37. Costello $\mathrm{AB}$, Osborne JW. Best practice in exploratory factor analysis: four recommendations for getting the most from your analysis. Practical Assessment Research \& Evaluation. 2005;10:1-9.

38. Watson D, Lee LA, Weber K, Assenheimer JS, Strauss ME, McCormick A. Testing the tripartite model: exploring the symptom structure of anxiety and depression in student, adult, and patient samples. J Abnorm Psychol. 1995; 104:15-25.

39. Jorge MR, Custódio O. Utilidade das escalas de avaliação para clínicos e pesquisadores. Rev Psiq Clín. 1999;26:102-5. 